\title{
Interactive Game Application of Cooking Malay Traditional Cuisines Toward Teenagers in Malaysia
}

\author{
Anis Najwa Rosli \& Ahmad Sofiyuddin Mohd Shuib* \\ Faculty of Art and Design, Universiti Teknologi MARA Perak Branch, Seri Iskandar Campus, 32610 \\ Seri Iskandar, Perak, MALAYSIA \\ *Corresponding author email: ahmad396@uitm.edu.my
}

Published: 28 September 2020

\begin{abstract}
The proposed interactive game application of cooking Malay traditional cuisines is a game designed to teach teenagers aged 12 to 24 years old in the cooking. There were a bunch of interactives cooking game applications on the internet, except for Malay cuisine games. This game is created to attract teenagers to making Malay traditional cuisine in interactive and fun ways. In this game, the user needs to follow the storyline in the game. Then, in the early game, they were given a list of ingredients to make some Malay cuisines. The player will be given the customer's requested food picture. Then the players need to click the right ingredients in making of the requested food by customer. If the player picks the wrong ingredients, then need to pick the ingredients from the start again. Next, this game concept is casual. With the time given, the players need to complete the order with minimum specific score to move forward to next stage. If they achieve the best result, the player will gain extra money and extra time bonus in the game. This game created with a different type of menus based on every chapter of the story. This game can attract teenagers to learn about cooking and the ingredients used in the food. This game is created to educate teenager yet entertain them at the same time.
\end{abstract}

Keywords: Malay Cuisine, Cooking Game, cooking learning, mobile application

eISSN: 2550-214X (C) 2020. The Authors. Published for Idealogy Journal by UiTM Press. This is an Open Access article distributed under the terms of the Creative Commons Attribution-NonCommercial-NoDerivatives License (http://creativecommons.org/licenses/by-nc-nd/4.0/), which permits non-commercial re-use, distribution, and reproduction in any medium, provided the original work is properly cited, and is not altered, transformed, or built upon in any way.

\section{INTRODUCTION}

Online games for cooking learning are available on the internet. Users can choose whatever they want to buy it, or simply download it from the website. Game-based learning to learn some new cooking recipes can have an impact on teenagers. It's because the game contains elements of animation and imagination that can stimulate the mind and creativity of teenagers (Ryan, 2018). Games are the favourite hobbies of the teenagers to make good of their leisure time. Not only does this game accommodate their leisure time, it is also a simple and immersive way to learn how to cook traditional cuisine.

\subsection{Problem Statements}

There are lots of games applications available on the internet. But there is no Malay traditional cooking. Most of the games that are available on the internet are mostly being adopted through to Western and Italian style cooking for example, "Good Pizza, Great Pizza", "Delicious World", "Cake Mania" and many more. There is no Malay cuisine game available yet on the internet. This is a good opportunity to expand the knowledge of traditional Malay cuisine worldwide by playing games.

Besides, there are bunch of cook book of Malay traditional cuisines that are provided at any book outlets. One of disadvantage about the cookbook is very complicated and full of text than image. So much text is really hard to understand by teenagers since it was their first time learning to cook new recipes. The good cook book must provide with lots of details and images of the menu's ingredients such as spices, vegetables, sauces, and many more. The less images of cooking and ingredients, the less 
teenagers' attraction to learn cooking. The images of ingredients and process of cooking is important (Chi, Chen, Chu, \& Lo, 2008).

Despite minimal preparation of ingredients in Malaysia's television cooking program somehow confounding teenagers who due to short show time are also unable to catch up with the preparation. Most of the program does not demonstrate a proper ingredient preparation tutorial. As we know, the program "MasterChef Junior" is an example of an interactive way to attract teenagers to cook with their own ideas of creativity.

\subsection{Objectives}

In developing this Cooking Game-based learning there are some objectives that would be achieved. It is hoped that list of objectives for this project will help to achieve the goal of this project. The objectives of this project are stated below:

a) To design a cooking game for teenagers.

b) To interact teenagers how to cook Malay foods in interactive way.

c) To use multimedia elements

\subsection{Significant of the Study}

This Learning Application is focused on interactive cooking tutorials for teenagers. It is because most of the game does not provide interactive ways to attract teenagers' attention to learn about Malay culture foods preparation. This study is to find the most teenagers' favourite and applicable interactive cooking game. Normally, some of the cooking games do not provide with Malay traditional food menus. The proposed project of interactive learning highlight factors is to make teenagers enjoy themselves yet learning at the same time. The researcher hopes this study can help and give benefit to the youth using this learning

\section{LITERATURE REVIEW}

This chapter of the literature review discusses immersive cooking game learning. The researcher also defines the related interactive learning literature in education such as the framework for mobile applications, media communication, style, and colour.

\subsection{The Advantages of Interactive Learning}

They're able to be open-minded in trying something new in the early stage of a child. The greatest effect on learning is the perception of sight and sound. Learning platform can improve the quality of digital media learning, and other resources can be a strong teaching tool (Ho, Chung, \& Lin, 2012). Interactive platforms can provide enjoyable learning environment (Morgan, 2010). Based on the learning process of multimedia (Mayer \& Clark, 2011), Images and pictures can help teenagers gain the awareness of details.

An interactive approach to modern methods can inspire teens to use traditional methods, (Terreni, 2010). These modern day, mobile application has grown rapidly over the years. (Nazruddin, 2012) says that mobile apps can be a powerful asset to the learning tool that allows teens to learn without any difficulty in places and times. This can have a major impact on adolescents ' education and entertainment. 


\section{METHODOLOGY}

This chapter, as shown in the title, contains the study's methodology on the game of Malay traditional cuisines. The researcher discusses in more depth the research technique, research process, research techniques, data collection methods, sample selection, research procedure, type of data analysis, ethical concerns, and project research constraints. The method used in this study is online surveys.

\section{FINDINGS AND DISCUSSION}

\subsection{Gender}

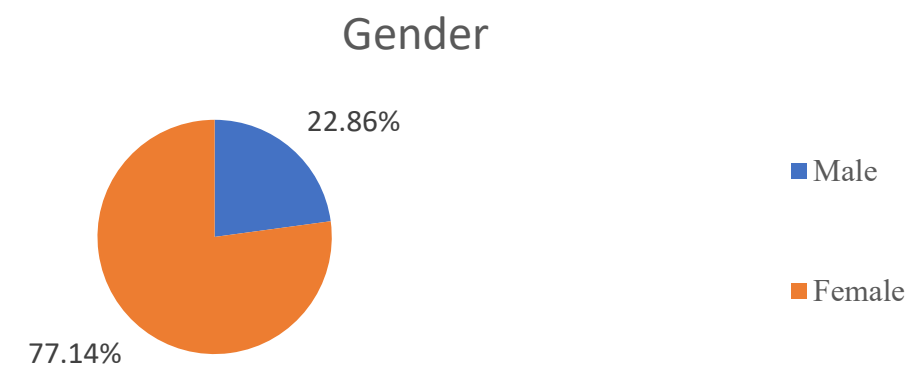

Figure 1: Percentage of gender

Based on Figure 1, there where total 35 respondents participated in this online survey, they are divided into two genders which female is the lead in the result with 27 participants and male with 8 participants.

\subsection{Age}

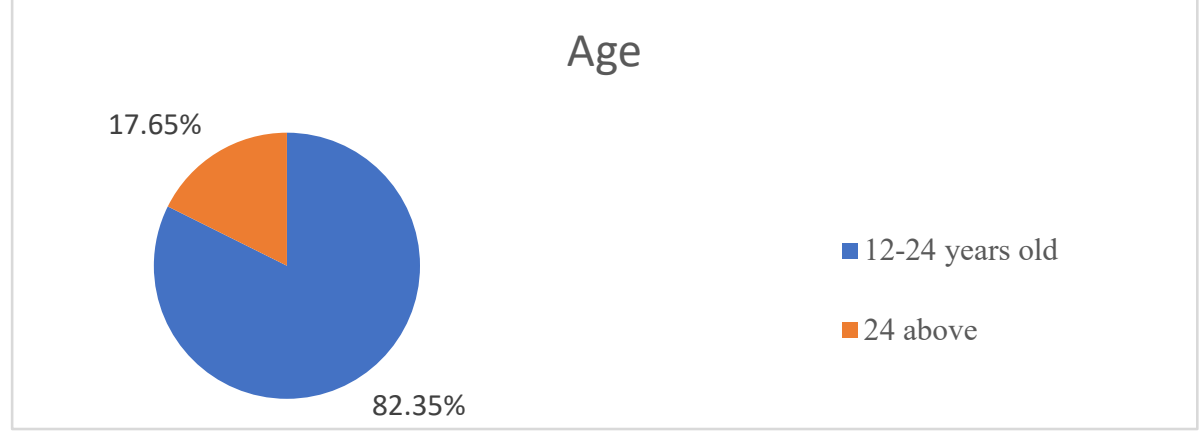

Figure 2: Percentage of age

Figure 2 recorded the participants' average age. There are 28 participants with an average age of 1224. Meanwhile, participants with adult average age 24 above are 8 people. 


\subsection{Food Type}

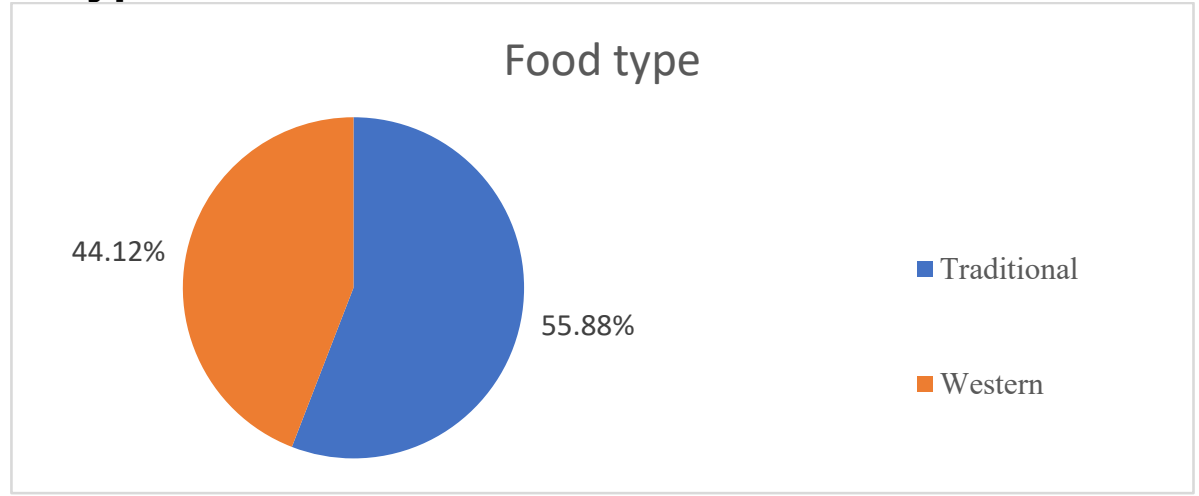

Figure 3: Percentage of food type

Based on Figure 3, 19 participants cannot cook Malay traditional foods and 15 participants cannot cook Western foods.

\subsection{Foods' Name}

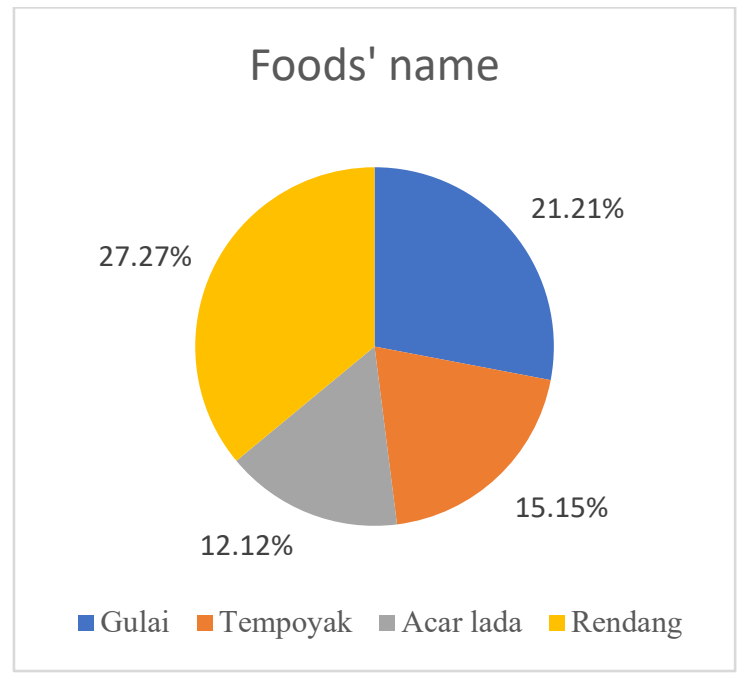

Figure 4: Percentage of foods' name

All the data on which kind of Malay traditional food that teenagers mostly don't know how to cook are recorded in Figure 4, rendang menu is the highest voting with a total of 12 participants. The second highest is tempoyak menu which was voted with a total of 8 participants. Meanwhile, the other 7 and 6 participants voted for gulai and acar lada. Sup is the less voted with no one voting for the menu. 


\subsection{Type of References}

Type of references

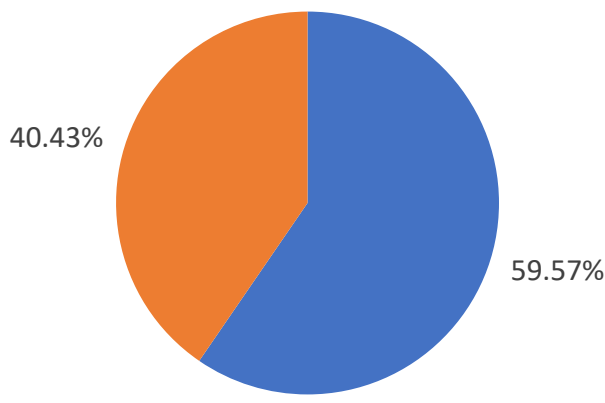

- Cookbook Cooking application

Figure 5: Percentage of type of references

Figure 5 recorded most of the teenager don't like to use cookbook instead using the cooking application. 28 participants don't like using cookbooks when learning the new recipes while 19 participants don't like using the cooking applications as a reference when learning the new recipes. Cookbook got the highest vote in this data.

\subsection{Reasons Answer Cook Book}

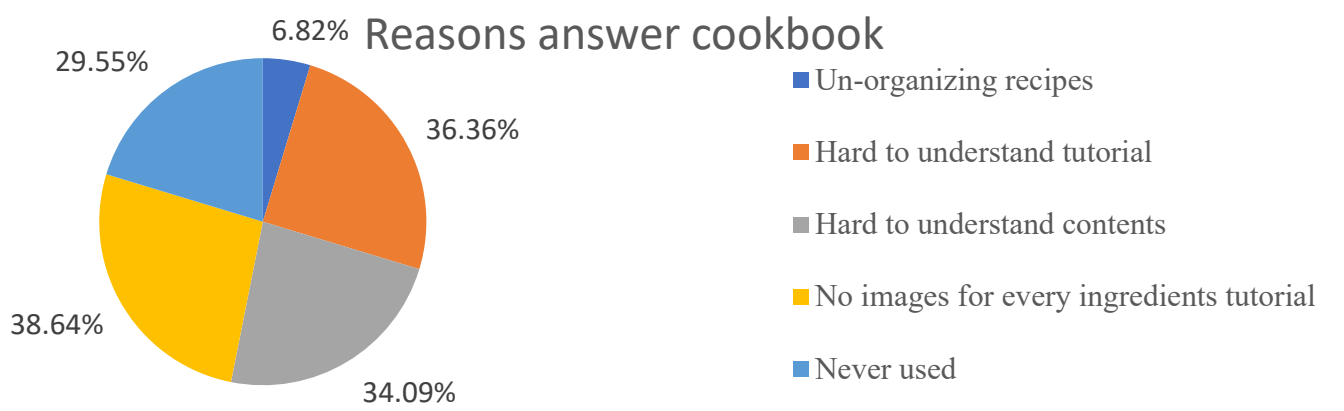

Figure 6: Percentage of reasons answer cookbook

Data from Figure 6 goes further detail in asking the respondents why they don't like using cook book rather than cooking application. These are what the respondents think about the disadvantage of the cookbook. The higher to the less vote from the participants are no images for every ingredients tutorial with a total of 17 voters, hard to understand the tutorial with the total of 16 voters, hard to understand contents with 16 voters, while 13 voters voted for never used cookbook, and lastly un-organizing recipes contents with 3 voters. 


\subsection{Logo Design}

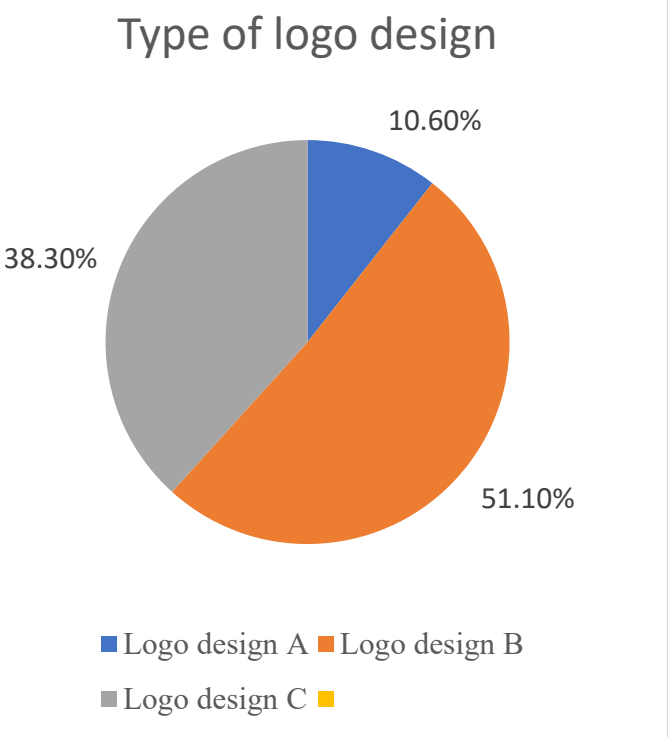

Figure 7: Percentage of logo design

Based on Figure 7, logo design B has the highest vote for the logo for The Malay Traditional Cuisine Game with 24 voters. The logo design A and logo design $C$ have a total of 5 and 18 voters.

\subsection{Characters Design}

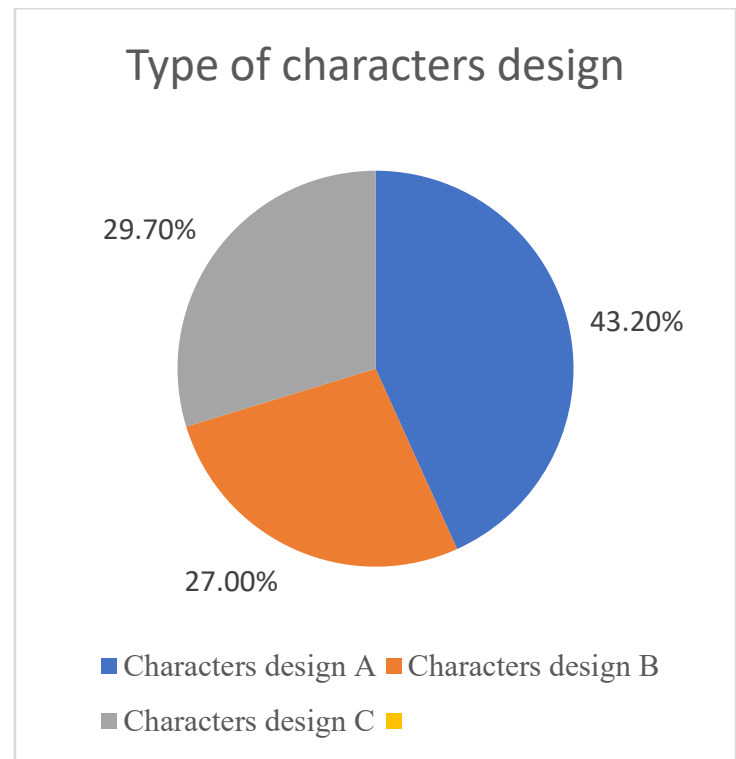

Figure 8: Percentage of characters design

For Figure 8, the question is about the voting of characters design concept. The question was prepared with 3 different design concept which is Characters Design A, Characters Design B, and Characters Design C. most voted for the character concept is Characters Design A with 16 voters. While for Character Design B and C voted by 11 and 10 participants. 


\subsection{Reason Answer Characters Design A}

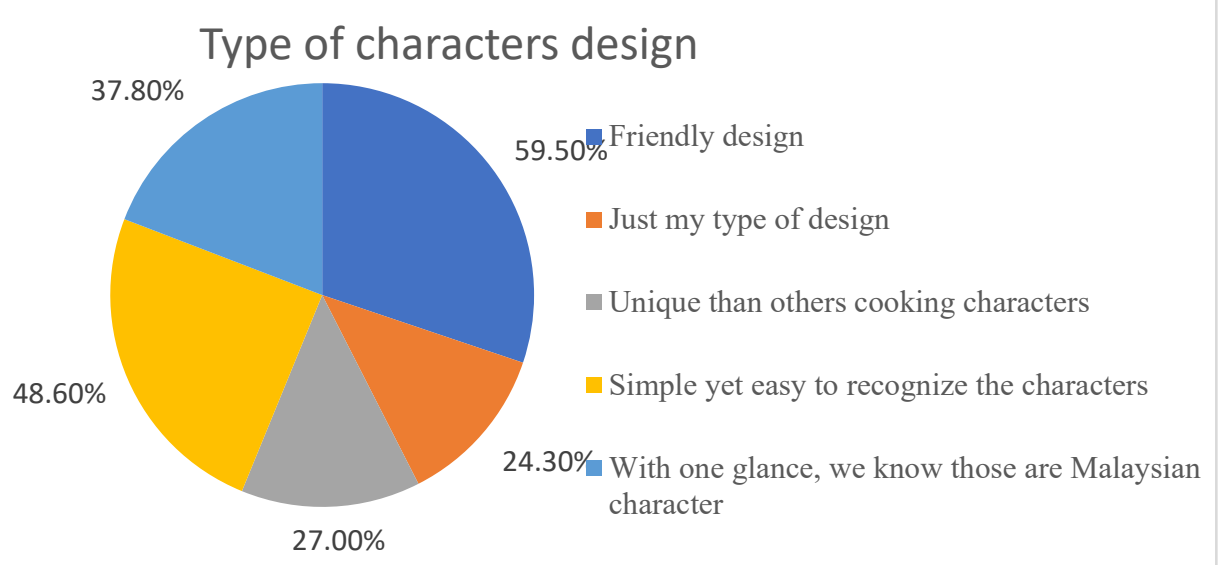

Figure 9: Percentage of answer characters design A

Figure 9 show the data of teenagers' view about the characters concept. They opinions about the design was 22 voted with friendly design, 18 voted for simple yet easy to recognize the characters, 14 voted for iconic Malaysian character, 10 voted for the unique design than others cooking characters, and 9 voted for their type of design concept.

\subsection{Background Design}

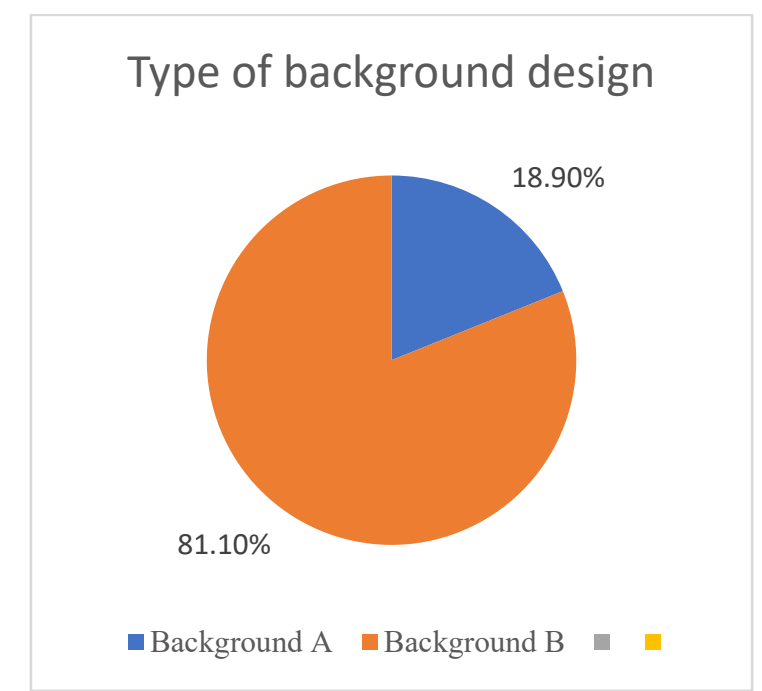

Figure 10: Percentage of background design

This question data about counter designs are shows in Figure 10. Counter design B style win for the most voted by teenagers with 30 voters, while counter design A voted by 7 teenagers. 


\subsection{Interface Design}

Type of interface design

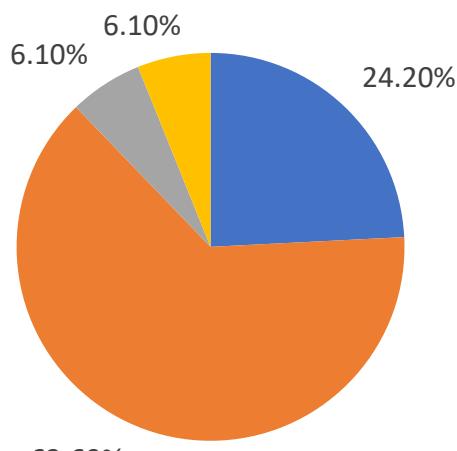

$63.60 \%$

- Interface A $\boldsymbol{\|}$ Interface B

- Interface C $\square$ Interface D

Figure 11: Percentage of interface design

Based on Figure 11, the question is about data of interface design in the game. Majority of the participants voted for interface B with 21 voters. Meanwhile, eight voters voted for interface A. Interface $\mathrm{C}$ and interface $\mathrm{D}$ have same total of voter which is 2 participants.

\subsection{Loading Animation Design}

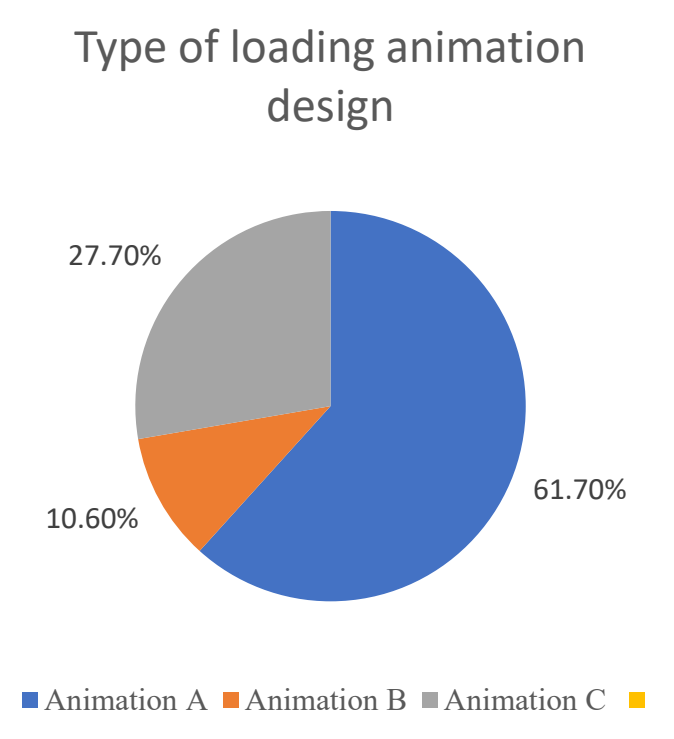

Figure 12: Percentage of loading animation design

For the last questionnaire, which is Figure 12 show the data of loading icon animation images. Animation A is the highest vote with 29 voters by teenagers. While, Animation B voted by teenagers with 13 votes and animation $C$ voted by teenagers with 5 votes. 


\section{CONCLUSION}

In the introduction section of this paper, the main objective of this project is to identify the factor that can lead an interactive way to interact with teenagers with the improvement of cooking skills. From the result of the inferential analysis, (refer to Figure 3) it shows that Traditional food leads the teenagers to suffer the lack of cooking skills.

The second objective of the project is aimed to create interactive ways of teaching cooking skills among teenagers. From figure 5, it is clear that most of the teenager doesn't like to use cookbook instead using cooking application when learning new recipes. Both objectives of the study have been achieved.

This study will focus more on the aspect of game designing, designing based on teenager preference, and how it will be implemented in the game layout design. Based on the analysis and data discovery, it can be concluded that this study has been able to establish. The guideline by TapBlaze, in designing the characters and Kak Yatie icon (ambassador of Adabi) that has been applied to create the game layout design is very effective and very adaptable to ensure a successful character design. 
Idealogy Journal

Volume 5 Issue 22020

溇 IDEALOGY

JOURNAL 